\title{
A Probabilistic Approach to Quantification of Melanin and Hemoglobin Content in Dermoscopy Images
}

\author{
Ali Madooei and Mark S. Drew \\ School of Computing Science \\ Simon Fraser University, Canada \\ \{amadooei, mark\}@cs.sfu.ca \\ http://www.cs.sfu.ca/ ramadooei
}

\begin{abstract}
We describe a technique that employs the stochastic Latent Topic Models framework to allow quantification of melanin and hemoglobin content in dermoscopy images. Such information bears useful implications for analysis of skin hyperpigmentation, and for classification of skin diseases. The proposed method outperforms existing approaches while allowing for more stringent and probabilistic modeling than previously.
\end{abstract}

\section{Introduction}

Computer-aided diagnosis of skin cancer is primarily based on analysis of clinical images of skin lesions, such as dermoscopy image 1 Interest in skin image analysis stems from the fact that useful visual information such as lesion colouration, pigmentation, etc. can be obtained via image processing in a computerized and automated fashion.

In this context, one less-studied but potentially interesting topic is quantification of skin's major chromophores (i.e. melanin and hemoglobin) from skin images, and its diagnostic utility. Such measurements are typically obtained through optical analysis of human skin via e.g. diffuse reflectance spectroscopy. This type of analysis is interesting as it offers the possibility of obtaining information about skin physiology and composition in a non-invasive manner. Such information may potentially be useful for diagnosis of skin diseases, screening the state of skin over the course of clinical therapy, and even final evaluation of efficacy of treatment. However, spectrophotometry (and similar techniques) require specific optical instruments. It would be economically and computationally beneficial if this information could be extracted from conventional clinical images. In particular, melanin and hemoglobin content of skin images can aid analysis of skin pigmentation, erythema, inflammation and hemodynamics.

Melanin and hemoglobin strongly absorb light in the visible spectrum and thus are major contributors to skin colouration. This motivates the hypothesis that, through analysis of skin colour, one may extract information about the underlying melanin and hemoglobin content. In this study, we make use of a recently published article by

\footnotetext{
${ }^{1}$ Dermoscopy is a popular [non-invasive] skin imaging technique. With the aid of optical magnification and cross-polarized lighting, it allows enhanced visualization of skin morphological characteristics which are often not discernible by the naked eye.
} 
Madooei et al. [1] which has explored this hypothesis. Their work extends the seminal work of Tsumura et al. [2] who employed independent component analysis (ICA) of skin images for extracting melanin and haemoglobin information. While Tsumura's goal was to develop a computationally efficient model of skin appearance for image synthesis, Madooei et al. employed the extracted information as a feature-space for supervised classification of classes Malignant vs. Benign 2

Independent component analysis is a powerful computational method that has been successfully used in many application domains but more specifically for signal processing aimed at solving the "blind source separation problem". It essentially defines a generative model of the observed data as linear mixtures of some underlying (hidden) factors. A variety of techniques have been proposed for this sort of data analysis which differ from each other on the merit of their assumptions, objective, and the constraints which they impose on the decomposition. These include factor analysis, principal component analysis (PCA), sparse component analysis, non-negative matrix factorization (NMF), projection pursuit, etc.

A somewhat related but separate class of techniques has emerged from the research in text/document analysis, known as latent topic models (LTM), which is aimed at identifying the underlying topics that affect the co-occurrence of words in a document. This is a probabilistic approach to model multivariate data (usually in form of histogram counts, i.e. probability distribution) by modeling the distributions of underlying (unobserved) statistical factors (topics) that affect them ${ }^{3}$. The two most popular techniques in this category are probabilistic latent semantic analysis (pLSA) and latent Dirichlet allocation (LDA). These language models (with their many variations) have gradually become an integral part of mainstream computer vision research, with a multitude of applications.

In this paper, we show that latent topic models can be equally effectively employed for quantification of melanin and hemoglobin content in skin lesion images. There are in fact many advantages to do so, as we will explain: Namely, latent topic models allow us to take advantage of their probabilistic framework. Also, as will be shown, the proposed model can overcome shortfalls of ICA-based models, in particular in terms of sign and scale ambiguity. We further demonstrate both qualitatively and quantitatively that probabilistic decomposition indeed results in a superior outcome compared to the stateof-the-art [1].

\section{Method}

As theoretical underpinning, we begin by adapting the well known dichromatic reflectance model [3] to describe the reflected light $L(\theta, \lambda)$ from skin surfaces:

$$
L(\theta, \lambda)=L_{\mathbb{S}}(\theta, \lambda)+L_{\mathbb{B}}(\theta, \lambda)
$$

\footnotetext{
${ }^{2}$ Another contribution of [1] over [2] is that a photometric model of image formation is utilized to account for and discard the effects of confounding factors in imagery such as light colour, intensity falloff, shading, and camera characteristics.

${ }^{3}$ This class of probabilistic modeling is more widely known as 'latent variable models' in the literature of mainstream statistical learning theory.
} 
where $L_{\mathbb{S}}$ (specular reflection) is the light reflected immediately at the skin surface, and $L_{\mathbb{B}}$ (body or diffuse reflection) is the radiance caused by the interaction of light with skin tissue. Here, $\lambda$ denotes wavelength and $\theta$ encapsulates geometrical dependencies. We can further expand this model to describe the body reflectance by optical properties of underlying skin layers, such as scattering and absorption of skin's major chromophores.

We consider a simplified skin tissue model composed of three layers from the top: epidermis, dermis and subcutis. The light scattering and absorption properties of epidermis and dermis depend mainly on the distribution and density of melanin and (total, i.e. oxy- \& reduced) hemoglobin, respectively 4 ; whereas the subcutis mainly contains fat cells which are assumed to diffuse all visible light [4].

By adapting a simple Lambert-Beer type of law for radiance from the multilayer skin tissue similar to that in [4], the body reflectance can be expressed as 5

$$
L_{\mathbb{B}}(\lambda) \simeq L_{e}(\lambda)+T_{e}(\lambda)^{2} L_{d}(\lambda)+T_{e}(\lambda)^{2} T_{d}(\lambda)^{2} L_{s}(\lambda)
$$

where $T_{e}$ and $L_{e}$ are the transmittance and diffuse reflectance of epidermis, respectively; $T_{d}$ and $L_{d}$ are those of the dermis; and $L_{s}$ is the diffuse reflectance of subcutis.

For dermoscopy images, the specular reflectance is suppressed by using crosspolarized lighting 6 Thus, $L_{\mathbb{S}} \simeq 0$. Also, it has been shown [5] that for $\lambda$ within the visible spectrum (400-700 nm), and for both fair and dark skins, the diffuse reflectance of epidermis and dermis layers are negligible compared to total diffuse reflectance. In other words, $L_{e, d} \ll L_{\mathbb{B}}$. If we assume, therefore, that $L_{e, d}$ both approach zero, then eq.(1) can be simplified as:

$$
L \simeq T_{e}^{2} T_{d}^{2} L_{s}
$$

The transmittance of the upper two layers of skin are assumed to bear an exponential relationship to the molar extinction coefficient of melanin and hemoglobin respectively [6] (assuming these are uniformly and randomly distributed, and their dimensions are much less than the thickness of the layers [7]):

$$
T_{e}=\exp \left(-d_{e} e_{m} C_{m}\right) \quad T_{d}=\exp \left(-d_{d} e_{h} C_{h}\right)
$$

where $d_{e}$ and $d_{d}$ are the thickness of epidermis and dermis respectively; $e_{m}$ is the extinction coefficient determined by the absorption spectrum of melanin; $C_{m}$ is the concentration of melanin; and accordingly, $e_{h}$ and $C_{h}$ are those quantities for hemoglobin.

By substituting eq. (4) in eq.(3) and taking the logarithm, we arrive at a linear equation which captures the relationship between the radiance entering the camera and the underlying composition of the colour pigments of skin:

$$
\log L(\theta, \lambda) \simeq m(\lambda) C_{m}(\theta)+h(\lambda) C_{h}(\theta)+l(\lambda)
$$

where we have lumped terms: $m(\lambda)=-2 d_{e} e_{m}(\lambda), h(\lambda)=-2 d_{d} e_{h}(\lambda)$, and $l(\lambda)=$ $\log L_{s}(\lambda)$. Note that the diffuse reflectance of subcutis is taken to be spatially uniform (i.e., $L_{s}$ is not a function of space).

\footnotetext{
${ }^{4}$ Note that scattering of collagen is ignored for simplicity.

${ }^{5}$ Here, reflection of each layer is considered separately and, for simplicity, interreflection inside each layer is ignored.

${ }^{6}$ Also, in general, the specular reflectance in clinical images of skin lesions can be attenuated by using photographic filters or image processing methods.
} 
Assuming a trichromatic colour camera with narrow-band and linear sensor response function $Q_{k \in\{R, G, B\}}(\lambda)=q_{k} \delta\left(\lambda-\lambda_{k}\right)$ [1], we can adapt eq.(5] to represent skin image data (N.B. for simplicity and without loss of generality we set $q_{k}=1$ ):

$$
\left[\begin{array}{l}
\log R(x, y) \\
\log G(x, y) \\
\log B(x, y)
\end{array}\right]=\left[\begin{array}{ll}
m\left(\lambda_{R}\right) & h\left(\lambda_{R}\right) \\
m\left(\lambda_{G}\right) & h\left(\lambda_{G}\right) \\
m\left(\lambda_{B}\right) & h\left(\lambda_{B}\right)
\end{array}\right]\left[\begin{array}{l}
C_{m}(x, y) \\
C_{h}(x, y)
\end{array}\right]+\left[\begin{array}{l}
l(\lambda) \\
l(\lambda) \\
l(\lambda)
\end{array}\right]
$$

Eq-6 can be recognized as an instance of the problem of blind source separation (BSS) where the observed data $(\log \mathbf{R}, \mathbf{G}, \mathbf{B})$ is described as linear mixtures of some unknown source signals $\left(\mathbf{C}_{m}\right.$ and $\left.\mathbf{C}_{h}\right)$, and the goal is to recover the source, using minimum assumptions on the mixing procedure (i.e., blindly). The mixing model in Eq-6 can be conveniently rewritten as $\mathbf{Y}=\mathbf{A S}$ where $\mathbf{Y} \in \mathbb{R}^{\mathbf{3} \times \mathbf{p}}$ is (mean-subtracted) image data ( $p=$ number of pixels); $\mathbf{S} \in \mathbb{R}^{\mathbf{2} \times \mathbf{p}}$ is the unknown source matrix which represents (relative) concentration of melanin and hemoglobin at each pixel; $\mathbf{A} \in \mathbb{R}^{\mathbf{3} \times \mathbf{2}}$ is the unknown mixing matrix which defines the contribution of each source in forming the observed data. We will call $\mathbf{S}$ the chromophores density matrix, and $\mathbf{A}$ the chromophores colour matrix. The goal of BSS is to recover these two quantities; however, the decomposition problem is underdetermined and additional assumptions are needed to solve it. Depending on what additional assumptions are considered, there are various methods for solving the BSS.

In [12], it is assumed the density of melanin and hemoglobin chromophores (i.e. $\mathbf{C}_{m}, \mathbf{C}_{h}$ ) are mutually independent over the image plane. Therefore, ICA [8] was applied to estimate chromophores density and colour matrices 8 . The empirical validity of this assumption is examined in [2], and its utility shown [1] to be useful for skin disease classification.

ICA finds the solution $\mathbf{S} \approx \mathbf{W Y}$ where $\mathbf{W}=\mathbf{A}^{+}$(where ${ }^{+}$denotes pseudoinverse; in case $\mathbf{A}$ is square and invertible, equalling $\mathbf{A}^{-1}$ ). In this notation, $\mathbf{W}$ is often referred to as unmixing or separating matrix. Within this framework, once $\mathbf{S}$ is estimated, it can be scaled; negated; and permuted with permutation matrix $\mathbf{P}$ and scaling matrix $\mathbf{M}$ such that new components remain independent: $\mathbf{S}^{\prime}=\mathbf{M P S}=\mathbf{M P W Y}$. So any matrix $\mathbf{W}^{\prime}=\mathbf{M P W}$ is also a valid separating matrix. This is often referred to as the inherent ambiguity in the ICA model (see Fig-1).

We now move forward from the deterministic nature of classical ICA algorithm to the stochastic treatment of latent topic models, which offers all the advantages of probabilistic frameworks while overcoming some of the limitation of the ICA model. In particular, we utilize probabilistic latent component analysis (PLCA) [9], an extension of the popular pLSA. Unlike ICA, which tries to characterize the observed data directly, PLCA characterizes the underlying distribution that generates the observed data.

We assume, without loss of generality, that observed data $\mathbf{Y}$ is actually a scaled probability distribution $P(x, y, k)_{k \in\{R, G, B\}}$ - or $P(\mathbf{x}, \mathbf{k})$ for brevity. To adapt the data into

\footnotetext{
${ }^{7}$ In eq. (6), the last term is a DC offset and can be easily removed by mean-subtraction.

${ }^{8}$ Note that our model is a more general treatment of image data compared to that in [1|2] where the dimension of observed data was reduced to the number of source signals.
} 


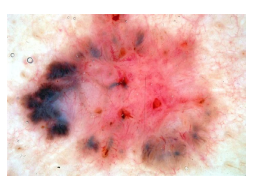

(a) Input

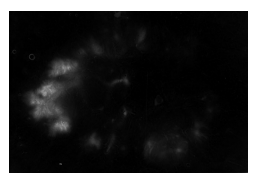

(b) Melanin

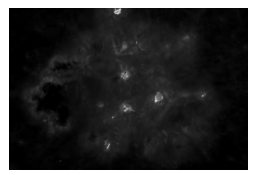

(c) Hemoglobin

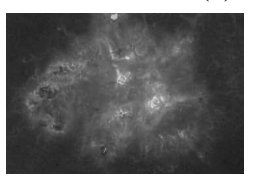

(d) IC-1

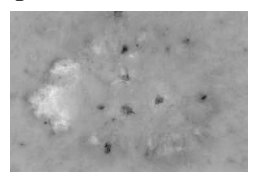

(e) $\mathrm{IC}-2$

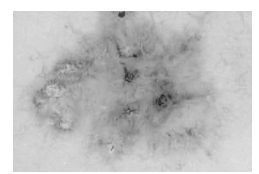

(f) $-\mathrm{IC}-1$

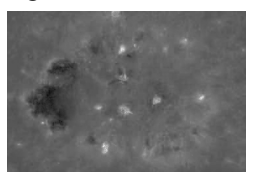

(g) -IC-2

Fig. 1. First row: PLCA output. Second row: output of ICA-based model in [1] showing ICA ambiguities (sign and permutation).

this assumption, we normalize $\mathbf{Y}$ to sum to unity 9 . The PLCA model considers a multivariate distribution as a mixture of a number of latent distributions that are specified by their marginals:

$$
P(\mathbf{x}, \mathbf{k})=\sum_{\mathbf{z}} P(\mathbf{z}) P(\mathbf{x} \mid \mathbf{z}) P(\mathbf{k} \mid \mathbf{z})
$$

where $\mathbf{Z}$ is the latent variable that indexes the hidden components, i.e. $z_{m}$ and $z_{h}$ for melanin and hemoglobin. The characteristic of eq.(7) is conditional independence, which fits well with the assumption of the ICA model. The goal of PLCA is to discover the marginals $P(\mathbf{x} \mid \mathbf{z})$ and $P(\mathbf{k} \mid \mathbf{z})$. These can be considered to be proportional to the probability distributions that generate chromophores density $\mathbf{S}$ and colour $\mathbf{A}$ matrices.

The estimation of marginals is achieved in a modified EM fashion: during the 'expectation' step, we estimate:

$$
P(\mathbf{z} \mid \mathbf{x}, \mathbf{k})=\frac{P(\mathbf{z}) P(\mathbf{x} \mid \mathbf{z}) P(\mathbf{k} \mid \mathbf{z})}{\sum_{\mathbf{z}} P(\mathbf{z}) P(\mathbf{x} \mid \mathbf{z}) P(\mathbf{k} \mid \mathbf{z})}
$$

followed by the 'maximization' step where we update the marginals using:

$$
\begin{gathered}
P(\mathbf{x} \mid \mathbf{z})=\frac{\sum_{\mathbf{k}} P(\mathbf{x}, \mathbf{k}) P(\mathbf{z} \mid \mathbf{x}, \mathbf{k})}{\sum_{\mathbf{x}} \sum_{\mathbf{k}} P(\mathbf{x}, \mathbf{k}) P(\mathbf{z} \mid \mathbf{x}, \mathbf{k})} \quad P(\mathbf{k} \mid \mathbf{z})=\frac{\sum_{\mathbf{x}} P(\mathbf{x}, \mathbf{k}) P(\mathbf{z} \mid \mathbf{x}, \mathbf{k})}{\sum_{\mathbf{x}} \sum_{\mathbf{k}} P(\mathbf{x}, \mathbf{k}) P(\mathbf{z} \mid \mathbf{x}, \mathbf{k})} \\
P(\mathbf{z})=\frac{\sum_{\mathbf{x}, \mathbf{k}} P(\mathbf{x}, \mathbf{k}) P(\mathbf{z} \mid \mathbf{x}, \mathbf{k})}{\sum_{\mathbf{x}, \mathbf{k}, \mathbf{z}} P(\mathbf{x}, \mathbf{k}) P(\mathbf{z} \mid \mathbf{x}, \mathbf{k})}
\end{gathered}
$$

Estimation of latent components in eq. (7) is obtained by iterating the above EM algorithm to convergence. The resulting decomposition can be expressed as a matrix factorization (cf. eq. (6)):

$$
\left[\begin{array}{l}
P\left(\mathbf{x}, k_{R}\right) \\
P\left(\mathbf{x}, k_{G}\right) \\
P\left(\mathbf{x}, k_{B}\right)
\end{array}\right]=\left[\begin{array}{ll}
P\left(k_{R} \mid z_{m}\right) & P\left(k_{R} \mid z_{h}\right) \\
P\left(k_{G} \mid z_{m}\right) & P\left(k_{G} \mid z_{h}\right) \\
P\left(k_{B} \mid z_{m}\right) & P\left(k_{B} \mid z_{h}\right)
\end{array}\right]\left[\begin{array}{cc}
P\left(z_{M}\right) & 0 \\
0 & P\left(z_{H}\right)
\end{array}\right]\left[\begin{array}{l}
P\left(\mathbf{x} \mid z_{m}\right) \\
P\left(\mathbf{x} \mid z_{h}\right)
\end{array}\right]
$$

\footnotetext{
${ }^{9}$ The scaling factor can be multiplied back into the estimated components to avoid e.g. quantization issues.
} 
From eq.111), we can define $\mathbf{C}_{m}=P\left(z_{m}\right) P\left(\mathbf{x} \mid z_{m}\right)$ and $\mathbf{C}_{h}=P\left(z_{h}\right) P\left(\mathbf{x} \mid z_{h}\right)$. It is obvious that these quantities are always positive and $\in[0,1]$ due to the nature of probability (thus we reduced ICA ambiguities). The non-negativity is a highly desired property, in particular when working with image data that is non-negative in nature. Also, the probabilistic outcome offers many advantages. For example, it allows easy integration with statistical learning algorithms; it enables one to evaluate the importance of an underlying factor; etc.

The probabilistic framework of PLCA can benefit from the abundant tools of statistical inference for estimation, in that it is possible to extend the model to account for various conditions depending on the problem in hand (some extensions are shiftinvariant PLCA [10], scale-invariant PLCA [11], sparse PLCA [10]). Moreover, it offers the ability to incorporate information known about the problem, or to impose hypothesized structure about the data by e.g. utilizing prior distribution during estimation. Obtaining this flexibility in non-stochastic models such as ICA, if possible, is complex and computationally expensive.

A potentially interesting utilization of PLCA model would be to control the order of separated components through e.g. the imposition of a priori probabilities. We plan to further investigate this. At this stage, however, we recover from permutation ambiguity by a simple post-processing: there is a high correlation between skin's Erythema-Index (redness) and the $\mathrm{a}^{*}$ channel of the CIE Lab colourspace [12]. The hemoglobin component, in a sense, embodies a similar meaning, i.e. redness of skin. Therefore, similarity to the $\mathrm{a}^{*}$ channel could be used as an indicator of hemoglobin distribution 11 .

\section{Experiments}

In order to verify the validity of the proposed skin colouration model, and the employed probabilistic decomposition approach, we need pathological data associated with skin images. For our dataset, such information is not available. Thus we rely on visual assessment: the proposed method appears to achieve superior results (compared to [1]) in highlighting and isolating hyperpigmented spots (e.g. Fig-1). Some sample outputs are shown in Fig-2.

We demonstrate the utility of our proposed method in application to skin disease classification. In keeping with [1], we applied a Logistic classifier to a set of 500 images taken from [13] with two classes consisting of malignant (melanoma and BCC) vs. all benign lesions (congenital, compound, dermal, Clark, Spitz and blue nevus; dermatofibroma; and seborrheic keratosis). For classification, the same set of features as in [1] are used (details are omitted for space considerations). Table 1 results are averaged over 10 runs of 10 -fold cross-validation. We see that our proposed method outperforms [1] in all measures, particularly for malignant lesions, where the results demonstrate up to $5 \%$ higher precision and recall for our method. Note that although this improvement is modest, our method achieves the separation of melanin and hemoglobin in a completely automatic fashion, whereas the components were manually labeled in [1].

\footnotetext{
${ }^{10}$ The similarity can be measured by e.g. computing the 2-D correlation coefficient.

${ }^{11}$ N.B. the outcome of the image analysis proposed here is different in general from results simply using CIE Lab values.
} 


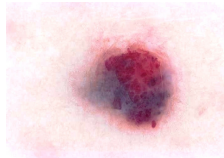

(a) Input

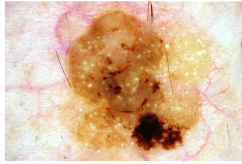

(d)

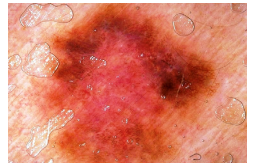

(g)

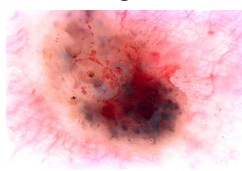

(j)

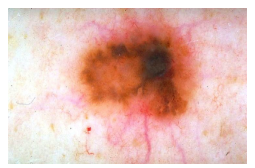

(m)

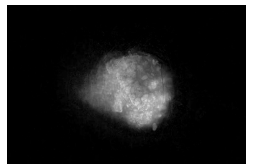

(b) Melanin

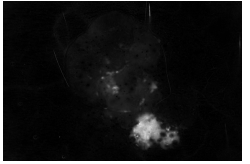

(e)

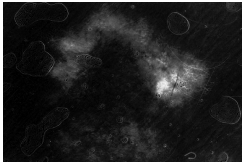

(h)

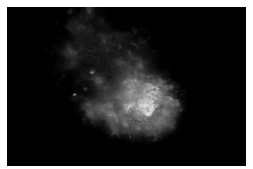

(k)

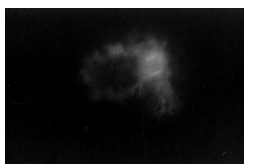

(n)

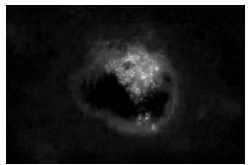

(c) Hemoglobin

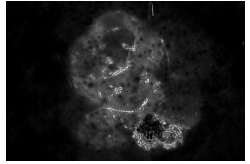

(f)

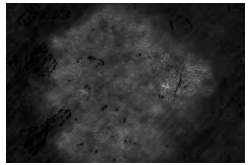

(i)

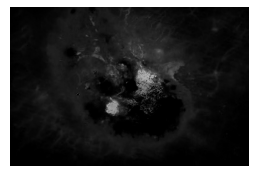

(1)

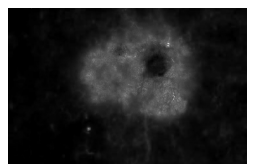

(o)

Fig. 2. Sample outputs - brighter means higher concentration

Table 1. Comparative Results of classifying the dataset in [1]. Since same feature-set \& classifier is used, the improvement is the result of using our proposed probabilistic decomposition. Note that Madooei et al. [1] also report classifying using RGB, HSV, and CIELAB colour spaces of which all are outperformed by their proposed colour feature space. We did not repeat those tests. Also, our result is averaged over 10 runs of 10 fold cross-validation. The std of weighted average is $0.043,0.044,0.046,0.041$ for precision, recall, $\mathrm{f}$-score, and area under the ROC curve (AUC) respectively.

\begin{tabular}{|c|c|c|c|c|c|c|}
\hline Colour Space & Class & $\mathbf{n}$ & Precision & Recall & F-measure & AUC \\
\hline \multirow{3}{*}{ Proposed Method } & Malignant & 135 & $\mathbf{0 . 8 5}$ & $\mathbf{0 . 8 3 7}$ & $\mathbf{0 . 8 4 3}$ & \multirow{3}{*}{} \\
\cline { 2 - 7 } & Benign & 365 & $\mathbf{0 . 9 4}$ & $\mathbf{0 . 9 4 5}$ & $\mathbf{0 . 9 4 3}$ & \multirow{0}{*}{.96} \\
\cline { 2 - 6 } & Weighted Avr. & 500 & $\mathbf{0 . 9 1 6}$ & $\mathbf{0 . 9 1 6}$ & $\mathbf{0 . 9 1 6}$ & \\
\hline \hline \multirow{3}{*}{ Method in [1] } & Malignant & 135 & 0.806 & 0.8 & 0.803 & \\
\cline { 2 - 6 } & Benign & 365 & 0.926 & 0.929 & 0.927 & 0.953 \\
\cline { 2 - 6 } & Weighted Avr. & 500 & 0.894 & 0.894 & 0.894 & \\
\hline
\end{tabular}




\section{Conclusion}

This paper highlights the use of latent topic models for medical image analysis. As a case study, we focused on quantification of skin's major chromophores (i.e. melanin and hemoglobin) from dermoscopy images. By developing a model of skin colouration, we formulated the undertaken task as a blind source separation problem. While prior art relies on independent component analysis (ICA), we applied probabilistic latent component analysis (PLCA) for discovery of sources. The probabilistic framework of PLCA offers many potential advantages over classical ICA. Moreover, when utilized for skin disease classification, the proposed approach outperformed ICA-based model as shown in our experiments.

For future work, we are interested in experimenting with larger datasets; as more data becomes available more ambitious problems can be tackled. Also, we would like to employ the LTM framework for the discovery of mid-level semantic visual concepts such as "asymmetric", "irregular", etc. to automatically annotate skin lesion images.

\section{References}

1. Madooei, A., Drew, M., Sadeghi, M., Atkins, M.: Intrinsic melanin and hemoglobin colour components for skin lesion malignancy detection. In: Ayache, N., Delingette, H., Golland, P., Mori, K. (eds.) MICCAI 2012, Part I. LNCS, vol. 7510, pp. 315-322. Springer, Heidelberg (2012)

2. Tsumura, N., Ojima, N., Sato, K., Shiraishi, M., Shimizu, H., Nabeshima, H., Akazaki, S., Hori, K., Miyake, Y.: Image-based skin color and texture analysis/synthesis by extracting hemoglobin and melanin information in the skin. ACM Trans. Graph. 22, 770-779 (2003)

3. Shafer, S.: Using color to separate reflection components. Color Research \& Application 10(4), 210-218 (1985)

4. Dawson, J., Barker, D., Ellis, D., Grassam, E., Cotterill, J., Fisher, G., Feather, J.: A theoretical and experimental study of light absorption and scattering by in vivo skin. Physics in Medicine and Biology 25(4), 695-709 (1980)

5. Wan, S., Parrish, J., Jaenicke, K.: Quantitative evaluation of ultraviolet induced erythema. Photochemistry and Photobiology 37(6), 643-648 (1983)

6. Diffey, B., Oliver, R., Farr, P.: A portable instrument for quantifying erythema induced by ultraviolet radiation. The British Journal of Dermatology 111(6), 663-672 (1984)

7. Takiwaki, H.: Measurement of erythema and melanin indices. In: Handbook of Non-Invasive Methods and the Skin, pp. 665-671. Informa Healthcare (1995)

8. Hyvarinen, A.: Fast and robust fixed-point algorithms for independent component analysis. IEEE TNN 10(3), 626-634 (1999)

9. Shashanka, M., Raj, B., Smaragdis, P.: Sparse overcomplete latent variable decomposition of counts data. In: NIPS, vol. 1, p. 2 (2007)

10. Smaragdis, P., Raj, B., Shashanka, M.: Sparse and shift-invariant feature extraction from non-negative data. In: ICASSP, pp. 2069-2072 (2008)

11. Hennequin, R., Badeau, R., David, B.: Scale-invariant probabilistic latent component analysis. In: IEEE WASPAA 2011, pp. 129-132 (2011)

12. Takiwaki, H.: Measurement of skin color: practical application and theoretical considerations. J. of Medical Investigation 44(3-4), 121-126 (1998)

13. Argenziano, G., Soyer, H., De Giorgio, V., Piccolo, D., Carli, P., Delfino, M., Ferrari, A., et al.: Interactive Atlas of Dermoscopy (Book and CD-ROM). Edra Medical Publishing \& New Media, Milan (2000) 